\title{
Clinical spectrum of neutropenia in children - analysis of 109 cases
}

\author{
Joanna Konieczek* (D), Natalia Bartoszewicz, Monika Richert-Przygońska, Ewa Charemska, \\ Edyta Węgrzyn, Anna Dąbrowska, Anna Urbańczyk, Elżbieta Grześk, Jan Styczyński, \\ Mariusz Wysocki, Sylwia Kołtan
}

Department of Pediatric Hematology and Oncology, Collegium Medicum in Bydgoszcz, Nicolaus Copernicus University in Toruń, Jurasz University Hospital 1, Bydgoszcz, Poland

\begin{abstract}
Introduction: The aim of this study was to retrospectively analyze the course of neutropenia in children hospitalized at a single pediatric hematology and oncology center, with particular emphasis on the assessment of risk factors for severe infectious complications.
\end{abstract}

Material and methods: The study included 109 children diagnosed with neutropenia unrelated to malignancy. The etiology, laboratory and genetic test results, and clinical data and course were analyzed.

Results: More than half (53.2\%) of the patients were ultimately diagnosed with benign childhood neutropenia. $74.5 \%$ of the children presented a chronic course of neutropenia, with a mean duration of 22 months. The duration of neutropenia had a significant impact on its clinical course: none of the patients with acute neutropenia had severe infections or required treatment. Among the patients with chronic neutropenia, a positive family history $(p<0.002)$, comorbidities $(p<0.005)$, severe infectious complications $(p<0.001)$ and the need for specific treatment $(p<0.004)$ were observed statistically more often in children with the congenital form of the disease.

Conclusions: Neutropenia in children usually has a benign course, but the prognosis largely depends on duration and etiology. History, clinical course, and ancillary test results should be carefully interpreted to identify patients with congenital neutropenia, due to the higher risk of complications and the need to treat patients in this group.

Key words: children, neutropenia unrelated to malignancy

Acta Haematologica Polonica 2021; 52, 5: 558-565

\section{Introduction}

Neutropenia is relatively common in children [1]. It is usually a symptom rather than a disease. Based on the absolute number of granulocytes (ANC), we classify neutropenia as being severe, moderate or mild. The duration of neutropenia can also be taken into account as a division criterion, allowing us to distinguish acute and chronic neutropenia [2]. Due to the pathomechanism of granulocyte count decrease, we can also distinguish primary from secondary neutropenia. In the case of primary neutropenia, a correct diagnosis can be challenging, but recently, thanks to the dynamic development of diagnostic techniques, clinicians have become more aware of the genetic background of the disease [3]. Depending on the type of neutropenia, different clinical variants are possible: from a frequently asymptomatic course in benign chronic childhood neutropenia (autoimmune or idiopathic background), to the
*Address for correspondence: Sylwia Kołtan, Department of Pediatrics, Hematology and Oncology, Ludwik Rydygier Collegium Medicum in Bydgoszcz, Nicolaus Copernicus University in Toruń, Jurasz University Hospital 1, Skłodowskiej-Curie 9, 85-094 Bydgoszcz, phone +48 5258548 61, fax +48525854867, e-mail: s.koltan@cm.umk.pl Received: 09.05.2021 Accepted: 06.06.2021

This article is available in open access under Creative Common Attribution-Non-Commercial-No Derivatives 4.0 International (CC BY-NC-ND 4.0) license, allowing to download articles and share them with others as long as they credit the authors and the publisher, but without permission to change them in any way or use them commercially.
PTHiT

Copyright (C) 2021

The Polish Society of Haematologists and Transfusiologists, Insitute of Haematology and Transfusion Medicine. All rights reserved. 
occurrence of frequent, serious and life-threatening infections in congenital neutropenia.

The following analysis aims to determine the characteristics of pediatric patients hospitalized for neutropenia, including specific groups separated by its etiology. This is a retrospective analysis of children diagnosed with neutropenia unrelated to disease and oncological treatment, hospitalized at a single pediatric hematology and oncology center between January 2005 and April 2020. Based on clinical data, laboratory and genetic test results, we wanted to analyze the clinical course, the risk of severe infectious complications, and the need for granulocyte colony-stimulating factor (G-CSF) treatment or antibiotic prophylaxis in patients from each group. We hope that our experience will bolster understanding about this heterogeneous group of patients and help to establish principles for the diagnosis and treatment of children with neutropenia.

\section{Material and methods}

\section{Patients}

The study included patients aged from 1 month to 18 years hospitalized due to neutropenia in the Department of Pediatrics, Hematology and Oncology of the Medical University Hospital No. 1 in Bydgoszcz, Poland. Neutropenia associated with neoplastic disease, myelodysplastic or myeloproliferative syndromes and their treatment was excluded from the study group.

\section{Clinical analysis}

The following data, obtained from medical records, was evaluated:

- basic patient characteristics such as gender, age at diagnosis of neutropenia, and duration of neutropenia;

- family history, with special attention paid to neutropenia and other hematological diseases, autoimmune diseases, and immune disorders;

- clinical data on course of neutropenia (i.e. frequency of infections, including severe infections such as sepsis; the presence of comorbidities);

- laboratory test results: blood count values at diagnosis, blood microscopic picture, serum levels of immunoglobulin major classes;

- other laboratory tests performed as necessary to further diagnosis, such as myelogram, presence of antigranulocytic antibodies, vitamin $\mathrm{B}_{12}$ and folic acid levels, chromosome fragility test with mitomycin;

- microbiological and serological tests for diagnosis of infection as a complication of neutropenia;

- any need for treatment with G-CSF or antibiotic prophylaxis.

\section{Genetic analysis}

Genetic testing was performed based on next-generation sequencing (NGS) techniques and molecular karyotyping. Detected point mutations or microdeletions were verified by testing the child's parents. Point mutations were additionally confirmed by Sanger sequencing.

\section{Definitions}

- Duration: up to 3 months: acute neutropenia, > 3 months: chronic neutropenia.

- ANC: agranulocytosis $(<100 / \mu \mathrm{L})$; severe neutropenia (100-500/ $\mu \mathrm{L})$; moderate neutropenia (500$-1,000 / \mu \mathrm{L})$; mild neutropenia <lower age limit but $>1,000 / \mu \mathrm{L}$.

- Etiology:

- congenital neutropenia (positive family history, diagnosis of genetically determined disease) with neutropenia as the only clinical manifestation and neutropenia as part of the disease syndrome;

- secondary neutropenia: autoimmune; secondary to infection, idiopathic.

\section{Classification}

A classification considering the etiology of neutropenia with definitions is shown in Table I.

\section{Principles of therapy}

Decisions to initiate treatment in children were made after analyzing the severity of neutropenia and the risk of complications. Treatment with G-CSF was initiated in patients with severe neutropenia or agranulocytosis who were at high risk for severe infections. In accordance with the recommendations of the Neutropenia Committee of the Marrow Failure Syndrome Group of the Associazione Italiana Emato-Oncologia Pediatrica (AIEOP), in children with severe congenital neutropenia the starting dose of G-CSF was $5 \mu \mathrm{g} / \mathrm{kg} /$ day [8]. In patients with secondary neutropenia, lower doses of G-CSF $(0.5-3 \mu \mathrm{g} / \mathrm{kg} /$ day $)$ were used [1]. Allogeneic hematopoietic stem cell transplantation (allo-HSCT) was used in children with congenital neutropenia who were unresponsive to G-CSF treatment with severe clinical course [8]. Antibiotic prophylaxis was used in children in whom infectious complications were observed, with a characteristic microorganism of known susceptibility to antibiotics used for prophylaxis.

\section{Statistical analysis}

Data analysis was performed using Statistica software for Windows version 10. Categorical variables were compared by chi-square test and non-categorical variables by Mann-Whitney $U$ test. A value of $p<0.05$ was considered statistically significant. 
Table I. Classification of neutropenia in children by etiology (acc. to [4-7])

\begin{tabular}{l|l}
\hline Diagnosis & Definition \\
\hline $\begin{array}{l}\text { Acute infection-induced neu- } \\
\text { tropenia }\end{array}$ & $\begin{array}{l}\text { Neutropenia secondary to infection, lasting up to three months. Decrease in granulocyte counts in } \\
\text { neutropenia secondary to infection can occur by a variety of mechanisms, including formation of an- } \\
\text { tigranulocytic antibodies or direct bone marrow suppression by toxins produced by pathogens }\end{array}$ \\
$\begin{array}{ll}\text { Post-infection persistent } \\
\text { neutropenia }\end{array}$ & $\begin{array}{l}\text { Neutropenia secondary to infection, lasting more than three months. Mechanism of onset as in acu- } \\
\text { te infection-induced neutropenia }\end{array}$ \\
$\begin{array}{l}\text { Benign chronic childhood } \\
\text { neutropenia }\end{array}$ & $\begin{array}{l}\text { Autoimmune or idiopathic neutropenia lasting 3+ months and characterized by benign clinical course. } \\
\text { Autoimmune neutropenia is diagnosed when serum autoantibodies directed against granulocytes are } \\
\text { detected. Idiopathic neutropenia is diagnosed when extensive diagnostic work-up fails to identify cause }\end{array}$ \\
$\begin{array}{ll}\text { Severe congenital neutro- } \\
\text { penia }\end{array}$ & $\begin{array}{l}\text { Severe neutropenia genetically determined, observed from birth, progressing with serious infectious } \\
\text { complications already in neonatal period and infancy. This is a group of diseases inherited in an au- } \\
\text { tosomal recessive (Kostmann syndrome, HAX1 gene mutation) manner and an autosomal dominant } \\
\text { (ELANE gene mutation) manner }\end{array}$ \\
$\begin{array}{l}\text { Specific genetic syndrome } \\
\text { associated with neutropenia }\end{array}$ & $\begin{array}{l}\text { Genetically determined diseases in which neutropenia occurs as part of complex syndromes invol- } \\
\text { ving multiple organ dysfunction (e.g. Shwachman-Diamond syndrome, WHIM, Fanconi anemia, etc.) }\end{array}$ \\
\hline \begin{tabular}{l} 
WHIM - warts, hypogammaglobulinemia, immunodeficiency, myelocathexis \\
\hline
\end{tabular}
\end{tabular}

\section{Results}

\section{Demographic analysis}

During the analysis period, neutropenia was diagnosed in 109 patients, comprising 65 (59.6\%) boys and 44 girls (40.4\%). The age at which the disease was diagnosed ranged from 1 month to 17 years, but most (71.6\%) of the patients were younger than 5 years. The mean age of the children at diagnosis was 49 months, with a median of 18 months. Agranulocytosis was observed in 23 patients (21.1\%), severe neutropenia in 46 (42.2\%), moderate neutropenia in 29 (26.6\%), and mild neutropenia in 11 (10.1\%).

\section{Clinical data}

Mild infections were the most common reason for diagnosis and blood counts on which neutropenia was diagnosed in 59 children (54.1\%). In 19 patients (17.4\%), neutropenia was found incidentally in an examination performed for another reason. In this group, as many as 12 children were diagnosed with idiopathic neutropenia. Severe infection, as the first manifestation and reason for neutropenia diagnosis, occurred in 10 children (9.2\%), of which congenital neutropenia was finally diagnosed in four.

The family history was eventful in 27 patients (24.8\%), of which neutropenia was also observed in 13 members of the immediate family (parents, siblings), and in the remaining 14 we observed autoimmune diseases, oncological diseases and other hematological disorders. Coexisting chronic diseases were found in 25 children (22.9\%).

\section{Diagnoses}

More than half of the children ( $n=58,53.2 \%$ ) were eventually diagnosed with benign chronic childhood neutropenia in the form of autoimmune ( $n=15,13.8 \%$ ) or idiopathic neutropenia ( $n=43,39.4 \%) .18$ (16.5\%) were diagnosed with acute infectious neutropenia, and another 14 (12.8\%) with persistent postinfectious neutropenia. In patients with neutropenia secondary to infection, the etiologic agent was identified in seven cases (one adenovirus infection, two parvovirus $B_{19}$ infections, two cytomegalovirus infections, one streptococcal angina, and one co-infection with adenovirus and parvovirus $\mathrm{B}_{19}$ ); urinary tract infection was diagnosed in another two patients, and acute otitis media in one. In the remaining children, respiratory tract infection was diagnosed without identification of the causative microorganism. Severe congenital neutropenia (SCN) was diagnosed in one patient. In another 14 children (12.8\%), certain genetic syndromes associated with neutropenia were diagnosed; this subgroup included such entities as Schwachmann-Diamond syndrome $(n=3)$, severe combined immunodeficiency (SCID) ( $n=3)$, autoimmune lymphoproliferative syndrome (ALPS) $(n=2)$, Fanconi anemia $(n=1)$, Pelger-Huet anomaly ( $n=1)$, transaldolase deficiency ( $n=1)$, WHIM (warts, hypogammaglobulinemia, immunodeficiency, myelocathexis) syndrome $(n=1)$, Noonan syndrome $(n=1)$, and Blackfan-Diamond syndrome $(n=1)$ (see Table II).

Two children were found to have vitamin B12 deficiency, and in another two the cause of neutropenia was undetermined. The characteristics of the patients in each group are set out in Table III.

The duration of neutropenia ranged from 1 week to 98 months, with a mean of 22 months and a median of 10 months. In the congenital neutropenia group, the mean duration was 41 months, and was statistically significantly higher compared to the other patients $(p<0.02)$.

Acute neutropenia was present in 27 patients (24.8\%). In this group, the majority were children with acute neutropenia with infection (Figure 1). None of the children in this group required G-CSF treatment or the inclusion of 
Table II. Characteristics of patients with congenital neutropenia

\begin{tabular}{|c|c|c|c|c|}
\hline Diagnosis* & $\mathbf{N}$ & Diagnosis based on & Age** & Therapy \\
\hline Schwachmann-Diamond syndrome & 3 & NGS - SBDS mutation & 2,$48 ; 120$ & G-CSF \\
\hline SCID & 3 & NGS - RAG1 mutation ( $\mathrm{n}=2$ ); ADA & $8 ; 3 ; 4$ & G-CSF, allo-HSCT \\
\hline ALPS & 2 & Clinical picture & $24 ; 84$ & G-CSF, sirolimus \\
\hline Fanconi anemia & 1 & Chromosome fragility test & 192 & - \\
\hline Pelger-Huet anomaly & 1 & No data available & 66 & - \\
\hline Transaldolase deficiency & 1 & Confirmed by genetic testing at another center & 2 & Antibiotic prophylaxis \\
\hline WHIM syndrome & 1 & NGS - CXCR4 mutation & 5 & - \\
\hline SCN & 1 & NGS - ELANE mutation & 1 & G-CSF, allo-HSCT \\
\hline Noonan syndrome & 1 & No data available & 60 & - \\
\hline Blackfan-Diamond syndrome & 1 & RPL35 deletion & 1 & - \\
\hline
\end{tabular}

Table III. Characteristics of study group according to diagnosed cause of neutropenia

\begin{tabular}{|c|c|c|c|c|c|}
\hline Parameter & Congenital & Chronic benign & $\begin{array}{l}\text { Acute infection- } \\
\text {-induced }\end{array}$ & $\begin{array}{l}\text { Post-infection } \\
\text { prolonged }\end{array}$ & Other \\
\hline \multirow[t]{2}{*}{ Number of patients } & $15 / 109$ & $58 / 109$ & $18 / 109$ & 14/109 & $4 / 109$ \\
\hline & $(13.8 \%)$ & $(53.2 \%)$ & $(16.5 \%)$ & $(12.8 \%)$ & $(3.7 \%)$ \\
\hline \multirow{2}{*}{$\begin{array}{l}\text { Age at diagnosis in months (mean; } \\
\text { min-max value) }\end{array}$} & 41 & 34 & 91 & 63 & 65 \\
\hline & $(1-192)$ & $(1-192)$ & $(7-199)$ & $(1-183)$ & $(2-204)$ \\
\hline \multirow[t]{2}{*}{ Male } & $9 / 15$ & $35 / 58$ & $12 / 18$ & $7 / 14$ & $2 / 4$ \\
\hline & $(60.0 \%)$ & $(60.3 \%)$ & $(66.7 \%)$ & (50.0\%) & $(50.0 \%)$ \\
\hline \multirow[t]{2}{*}{ Female } & $6 / 15$ & $23 / 58$ & $6 / 18$ & $7 / 14$ & $2 / 4$ \\
\hline & $(40.0 \%)$ & $(39.7 \%)$ & (33.3\%) & $(50.0 \%)$ & $(50.0 \%)$ \\
\hline \multirow[t]{2}{*}{ ANC $<500 / \mu \mathrm{L}$} & $11 / 15$ & $43 / 58$ & $6 / 18$ & $5 / 14$ & $3 / 4$ \\
\hline & $(73.3 \%)$ & (74.1\%) & (33.3\%) & $(35.7 \%)$ & $(75.0 \%)$ \\
\hline Duration in months (mean) & 41.3 & 26.2 & 1.3 & 14.2 & 3.4 \\
\hline \multirow[t]{2}{*}{ Transient nature } & $8 / 15$ & $53 / 58$ & $18 / 18$ & $14 / 14$ & $4 / 4$ \\
\hline & (53.3\%) & $(91.4 \%)$ & $(100 \%)$ & $(100 \%)$ & $(100 \%)$ \\
\hline \multirow[t]{2}{*}{ Eventful family history } & $9 / 15$ & $11 / 58$ & $2 / 18$ & $4 / 14$ & $1 / 4$ \\
\hline & $(60.0 \%)$ & $(19.9 \%)$ & $(11.1 \%)$ & $(28.6 \%)$ & $(25 \%)$ \\
\hline \multirow[t]{2}{*}{ Infections } & $13 / 15$ & $41 / 58$ & $4 / 18$ & $1 / 14$ & $0 / 4$ \\
\hline & $(86.7 \%)$ & $(70.7 \%)$ & $(22.2 \%)$ & $(7.1 \%)$ & $(0 \%)$ \\
\hline \multirow[t]{2}{*}{ Severe infections } & $9 / 15$ & $5 / 58$ & $0 / 18$ & $1 / 14$ & $0 / 4$ \\
\hline & $(60.0 \%)$ & $(8.6 \%)$ & $(0.0 \%)$ & $(7.1 \%)$ & $(0 \%)$ \\
\hline \multirow[t]{2}{*}{ Coexisting immunodeficiency } & $14 / 15$ & $15 / 58$ & $1 / 18$ & $3 / 14$ & $1 / 4$ \\
\hline & $(93.3 \%)$ & (25.9\%) & $(5.6 \%)$ & $(21.4 \%)$ & $(25.0 \%)$ \\
\hline \multirow[t]{2}{*}{ Coexisting chronic diseases } & $9 / 15$ & $12 / 58$ & $4 / 18$ & $1 / 14$ & $0 / 4$ \\
\hline & $(60.0 \%)$ & $(20.7 \%)$ & $(22.2 \%)$ & $(7.1 \%)$ & $(0 \%)$ \\
\hline \multirow[t]{2}{*}{ Treatment } & $8 / 15$ & $15 / 58$ & $0 / 18$ & $1 / 14$ & $0 / 4$ \\
\hline & (53.3\%) & $(25.9 \%)$ & $(0 \%)$ & $(7.1 \%)$ & $(0 \%)$ \\
\hline
\end{tabular}




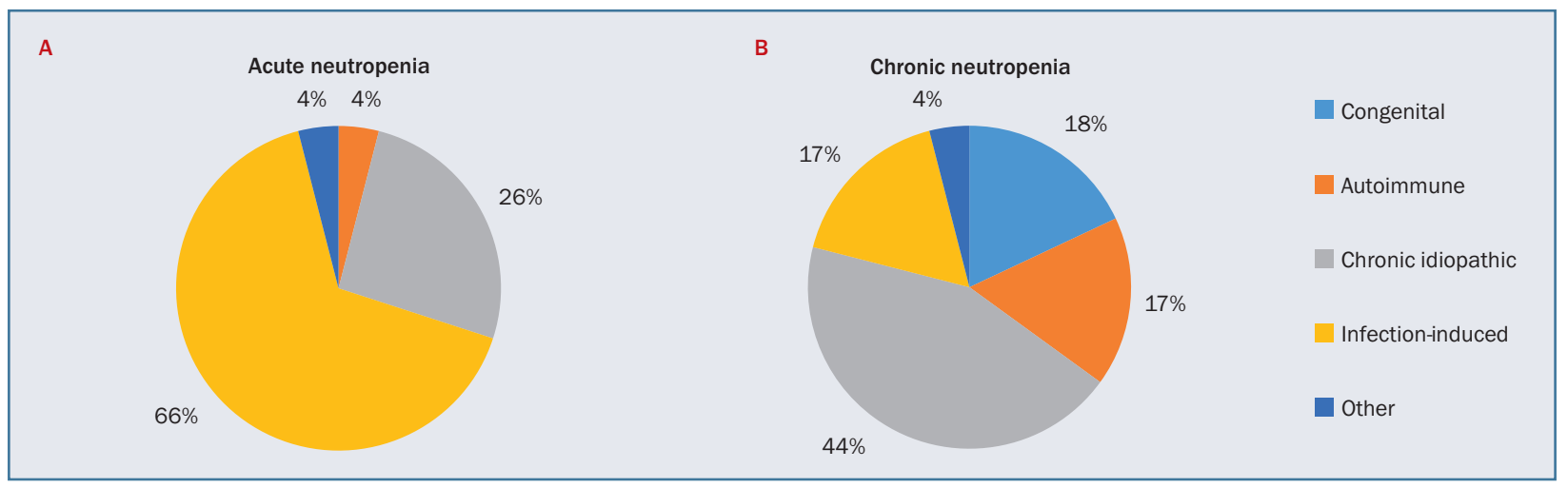

Figure 1A, B. Causes of acute and chronic neutropenia

antibiotic prophylaxis. Severe infections requiring hospitalization did not occur in any child during the follow-up period.

Chronic neutropenia was found in the remaining $82 \mathrm{pa}-$ tients, representing $74.5 \%$ of the children. Benign chronic neutropenia in children was the most common finding (Figure 1). Twenty-four children (29.3\% of patients with chronic neutropenia) required treatment. Of these, four with congenital neutropenia underwent an allo-HSCT procedure. Among the patients with chronic neutropenia, its transient nature was observed in 70 children (85.4\%).

\section{Clinical course}

During the observation period, infections occurred in 76 children (69.7\%) in the whole study group; these were mostly mild infections typical for childhood. Serious infections were found in 23 children (21.1\%), of whom four with congenital neutropenia had multiple, recurrent, and complicated life-threatening infections. Serious infections were statistically significantly more common in children with congenital neutropenia ( $p<0.001$ ); these patients also had an almost ten-fold increased risk of sepsis during the follow-up period compared to other patients (Table IV).

Patients with benign chronic childhood neutropenia had a similar clinical course, regardless of the etiology of neutropenia (autoimmune vs idiopathic). In the comparative analysis of the two groups there were no statistically significant differences in parameters such as age of child at diagnosis $(p<0.75)$, duration of neutropenia $(p<0.79)$, percentage of children with severe neutropenia and agranulocytosis at diagnosis ( $p<0.44$ for agranulocytosis and $p<0.65$ for severe neutropenia, respectively), or occurrence of severe infections $(p<0.65)$. The only statistically significant difference between the two groups was the percentage of children in whom the decision to initiate G-CSF treatment was made (53.3\% of patients with autoimmune neutropenia and $16.3 \%$ with idiopathic neutropenia, respectively, $p<0.005$ ).

\section{Treatment}

In 23 patients (21.1\%), the decision was made to initiate treatment in the form of antibiotic prophylaxis or G-CSF treatment. In three of them, antibiotic prophylaxis alone was used, in 19 G-CSF therapy alone, and in one patient both forms of treatment were implemented.

In three patients, amoxicillin was used for antibiotic prophylaxis, in one case in combination with cotrimoxazole administered twice a week, and in one boy trimethoprim was used.

G-CSF treatment was used in children at high risk of severe infectious complications (patients with chronic severe neutropenia or agranulocytosis, with pre-existing severe infections). For benign chronic childhood neutropenia, both autoimmune and idiopathic, lower doses of G-CSF $(0.5-3 \mu \mathrm{g} / \mathrm{kg} /$ day) were used and were usually sufficient to achieve an increase in granulocyte count $>1,000 / \mu \mathrm{L}$ that was sustained after treatment. Children with congenital neutropenia were more than five times more likely to require treatment than other patients (Table IV), and treatment with G-CSF was usually not satisfactory, despite daily administration and higher doses ( $5 \mu \mathrm{g} / \mathrm{kg}$ initially, multiple higher doses in case of no response). In four children with congenital neutropenia (three with severe combined immunodeficiency (SCID) and one with severe congenital neutropenia associated with ELANE gene mutation), an allogeneic blood stem cell transplantation procedure was performed, because this was the only therapeutic option leading to a cure. All patients achieved disease remission after transplantation, but patients with SCID required intravenous immunoglobulin (IVIG) supplementation due to persistent hypogammaglobulinemia after transplantation. A boy with neutropenia due to ELANE gene mutation despite normalization of blood morphology parameters after allogeneic hematopoietic stem cell transplantation (allo-HSCT) died due to post-transplant complications. 
Table IV. Odds ratio of diagnosis of congenital neutropenia and other neutropenia for selected data of family history, duration of neutropenia, clinical course of neutropenia

\begin{tabular}{|l|c|c|c|}
\hline Parameter to be assessed & OR & $\begin{array}{c}\text { Confidence } \\
\text { of OR (-95\%; 95\%) }\end{array}$ & $P$ value \\
\hline Male sex & 1.018 & $0.335 ; 3.095$ & 0.9751 \\
\hline Transient nature of neutropenia & 0.078 & $0.021 ; 0.288$ & 0.0001 \\
\hline Eventful family history & 6.333 & $1.998 ; 20.077$ & 0.0017 \\
\hline Infections & 3.683 & $0.784 ; 17.303$ & 0.0986 \\
\hline Fever & 1.761 & $0.589 ; 5.266$ & 0.3115 \\
\hline UTI & 4.450 & $0.942 ; 21.031$ & 0.0596 \\
\hline Acute otitis media & 1.453 & $0.282 ; 7.488$ & 0.6551 \\
\hline Sepsis & 8.900 & $2.192 ; 36.142$ & 0.0022 \\
\hline Coexisting chronic diseases & 5.176 & $1.652 ; 16.223$ & 0.0048 \\
\hline Need for treatment & 5.571 & $1.767 ; 17.564$ & 0.0034 \\
\hline Antibiotic prophylaxis & 7.077 & $0.916 ; 54.656$ & 0.0606 \\
\hline G-CSF treatment & 1.915 & 0.5386 .824 & 0.3162 \\
\hline
\end{tabular}

Values in bold are statistically significant; OR - odds ratio; UTI - urinary tract infection; G-CSF - granulocyte colony-stimulating factor

\section{Discussion}

Neutropenia is relatively common in children, but is usually secondary in nature, with remission occurring during the follow-up period. Hospitalization in children with neutropenia is necessary if infectious complications develop or if the diagnosis is broadened, especially when congenital neutropenia is suspected.

To the best of our knowledge, this study is the largest single-center analysis of pediatric patients with neutropenia in Poland. In order to identify at-risk patients, a variety of methods was used in the diagnostic process, including modern genetic diagnostic techniques such as NGS, which also enabled the diagnosis of rare and ultra-rare diseases developing alongside neutropenia.

On the basis of the analysis of the presented data, it can be concluded that in most cases neutropenia was characterized by a mild course, and in more than half of the patients spontaneous remission was observed within the first year of follow-up. The group of patients with congenital neutropenia was the most vulnerable to infectious complications. They were characterized by a statistically significantly higher risk of severe infections and the need for treatment. Children from this group were more frequently affected by coexisting chronic diseases and a positive family history, and more often the first manifestation of neutropenia was severe infection.

The presented observations are consistent with reports from other centers [2]. The duration of neutropenia had a significant impact on its clinical course, regardless of the severity of neutropenia at the time of diagnosis. In children with acute neutropenia, severe infections were not observed, nor did they require treatment.
The largest group consisted of children with benign chronic childhood neutropenia. This group included both children with autoimmune and idiopathic neutropenia. Because of the similar clinical course, some authors have suggested that idiopathic neutropenia may in fact have an autoimmune basis [3]. This is supported by the fact that only in $60-74 \%$ of patients with autoimmune neutropenia are antigranulocytic antibodies detected $[9,10]$. This may be caused by too low titers of antibodies, not exceeding the detection threshold of the method, or by too long an interval between the appearance of antibodies and the test, when a decrease in ANC resulting from autoimmunity is observed, but autoantibodies are not detected [11].

The only statistically significant difference between our two groups of patients was the percentage of children who received G-CSF treatment. Apart from that, no statistically significant differences were observed in terms of clinical course or main parameters describing neutropenia.

The smallest group consisted of children with congenital neutropenia. It is worth underscoring that congenital neutropenia is an extremely rare disorder, which means that the diagnosis is often made late. Another factor complicating the diagnosis is the fact that the group of diseases classified as congenital neutropenia is very heterogeneous and may manifest with the onset of neutropenia at an older age. In our analysis, we also observed great heterogeneity in the group of patients with congenital neutropenia, ranging from severe congenital neutropenia based on ELANE gene mutation, through neutropenia coexisting with primary immunodeficiency (SCID), children with diseases manifesting in infancy (Swachmann-Diamond syndrome, Fanconi anemia), as well as children with congenital neutropenia of mild course (Pelger-Huet anomaly). 
One patient in this group was diagnosed with warts, hypogammaglobulinemia, immunodeficiency, myelocathexis (WHIM) syndrome, an extremely rare genetically determined disease with neutropenia that had only been described in 105 patients worldwide up to 2019 [12]. This syndrome is caused by a mutation of the CXCR4 receptor, inherited in an autosomal dominant manner. Neutropenia in WHIM syndrome is due to myelocatexia, a phenomenon involving retention of mature granulocytes in the bone marrow [12, 13]. It is noteworthy that in the described patient, the clinical course was uncharacteristic (absence of viral warts), and only thanks to NGS were we able to establish the diagnosis. This is an excellent illustration of the fact that in rare and ultra-rare diseases, it is often only thanks to modern genetic tests that a diagnosis can be made [14].

Children with neutropenia secondary to infection, both acute and persistent, had the mildest clinical course. In this group, only one patient developed severe infections requiring hospitalization during the follow-up period. This confirms previous observations that neutropenia secondary to infection is characterized by an uncomplicated course due to, among other things, its usually episodic nature, the older age of children, and the rare coexistence of chronic diseases (only in $15.6 \%$ of children with neutropenia secondary to infection) [5, 15-17].

Interestingly, drug-induced neutropenia was not observed in any of the hospitalized patients, which contradicts data from other centers $[2,18]$. This is probably due to the fact that children under immunological care for drug-induced neutropenia usually do not require hospitalization because the cause of the decrease in neutrophil count is known, and the treatment of choice is drug switching, dose reduction or withdrawal [19].

In the study group, treatment was initiated in children with a history of severe infections or in patients with neutropenia associated with a high risk of serious infectious complications.

In children with severe congenital neutropenia, the starting dose of G-CSF was $5 \mu \mathrm{g} / \mathrm{kg} /$ day, with doses increased as needed [8], but despite high doses of G-CSF satisfactory ANC levels were not always achieved. When treatment was responded to, the increase in granulocyte counts did not necessarily translate into clinical improvement in this group, probably due to pre-existing severe infections and their complications.

Benign chronic childhood neutropenia, whether autoimmune or idiopathic, has traditionally been treated with lower doses of G-CSF (0.5-3 $\mu \mathrm{g} / \mathrm{kg} /$ day) and this has usually been sufficient to achieve an increase in granulocyte count $>1,000 / \mu \mathrm{L}$. In our group of patients, an initial dose of $5 \mu \mathrm{g} / \mathrm{kg}$ body weight three times per week was used and then the lowest effective dose to be used two or three times per week was sought. This treatment was usually initiated in children with previous serious infections, was usually well tolerated, and led to a satisfactory response and a decrease in infectious complications. As mentioned above, decisions to start G-CSF treatment were the only statistically significant difference between children with autoimmune and idiopathic neutropenia, with one in two children with autoimmune neutropenia, and one in six with idiopathic neutropenia, choosing to do so. This is probably due to concerns that the administration of G-CSF will disturb the clinical picture and the results of laboratory tests where there is a need to broaden or repeat the diagnostics in patients diagnosed with idiopathic neutropenia.

In the present group, blood stem cell transplantation was performed in three patients with SCID and one with $\mathrm{SCN}$. All of these children had severe, life-threatening infections requiring several weeks of hospitalization in early childhood. Among these patients, the three with SCID achieved remission of the underlying disease and withdrawal of neutropenia, whereas the boy with SCN died due to post-transplant complications.

Antibiotic prophylaxis was used in individual patients, always after careful consideration of the pros and cons of such an approach. It was implemented in children with recurrent infections with a specific pathogen, and was maintained in cases of good clinical response.

\section{Conclusions}

In conclusion, based on the data analyzed, the occurrence of severe infections was more likely in children with neutropenia lasting more than three months, and children with congenital neutropenia were most at risk. An eventful family history, the coexistence of other chronic diseases, and the persistent nature of neutropenia, as well as the occurrence of severe infectious complications, also as the first manifestation of neutropenia: these features characterized patients who were eventually diagnosed with congenital neutropenia.

The mildest course was observed in children with neutropenia secondary to infection, which was also characterized by rapid, spontaneous remission.

Based on the above analysis, it is clearly vital in the diagnosis of children with neutropenia to take a thorough history and to evaluate laboratory findings in conjunction with the child's clinical assessment. Neutropenia itself is only an abnormality in laboratory tests, and doctors' experience and knowledge are crucial in assessing the risk of complications associated with it, and in deciding on both in-depth diagnostics and treatment.

\section{Authors' contributions}

JK - data collection and interpretation, statistical analysis, literature collection and description of results. NB, MR-P, EC, EW, AD, AU, EG - data collection and interpretation. JS, MW - critical review for important intellectual 
content. SK - thesis draft, critical review for important intellectual content, acceptance of final version for publication.

\section{Conflict of interest}

The authors declare no conflict of interest.

\section{Financial support}

None.

\section{Ethics}

The work described in this article has been carried out in accordance with The Code of Ethics of the World Medical Association (Declaration of Helsinki) for experiments involving humans; EU Directive 2010/63/EU for animal experiments; Uniform Requirements for manuscripts submitted to Biomedical journals.

\section{References}

1. Dale DC. How I manage children with neutropenia. Br J Haematol. 2017; 178(3): 351-363, doi: 10.1111/bjh.14677, indexed in Pubmed: 28419427.

2. Karapinar TH, Oymak Y, Ay Y, et al. Chronic neutropenia in childhood: experience from a single center. J Pediatr Hematol Oncol. 2016; 38(1): e35-e38, doi: 10.1097/MPH.0000000000000449, indexed in Pubmed: 26479986.

3. Lindqvist $\mathrm{H}$, Carlsson $\mathrm{G}$, Moell J, et al. Neutropenia in childhood: a 5-year experience at a tertiary center. Eur J Pediatr. 2015; 174(6): 801-807, doi: 10.1007/s00431-014-2465-5, indexed in Pubmed: 25435257.

4. Schwartzberg LS. Neutropenia: etiology and pathogenesis. Clin Cornerstone. 2006; 8(Suppl 5): S5-S11, doi: 10.1016/s10983597(06)80053-0.

5. Sheen JM, Kuo HC, Yu HR, et al. Prolonged acquired neutropenia in children. Pediatr Blood Cancer. 2009; 53(7): 1284-1288, doi: 10.1002/pbc.22247, indexed in Pubmed: 19711442.

6. Chung BHY, Chan GCF, Lee TL, et al. Chronic benign neutropenia among Chinese children. Hong Kong Med J. 2004; 10(4): 231-236, indexed in Pubmed: 15299167.

7. Segel GB, Halterman JS. Neutropenia in pediatric practice. Pediatr Rev. 2008; 29(1): 12-23; quiz 24, doi: 10.1542/pir.29-1-12, indexed in Pubmed: 18166617.
8. Fioredda F, Calvillo M, Bonanomi S, et al. Neutropenia Committee of the Marrow Failure Syndrome Group of the AIEOP (Associazione Italiana Emato-Oncologia Pediatrica). Congenital and acquired neutropenias consensus guidelines on therapy and follow-up in childhood from the Neutropenia Committee of the Marrow Failure Syndrome Group of the AIEOP (Associazione Italiana Emato-Oncologia Pediatrica). Am J Hematol. 2012; 87(2): 238-243, doi: 10.1002/ajh.22242, indexed in Pubmed: 22213173.

9. Bux J, Behrens G, Jaeger G, et al. Diagnosis and clinical course of autoimmune neutropenia in infancy: analysis of 240 cases. Blood. 1998; 91(1): 181-186, indexed in Pubmed: 9414283.

10. Bruin M, Dassen A, Pajkrt D, et al. Primary autoimmune neutropenia in children: a study of neutrophil antibodies and clinical course. Vox Sang. 2005; 88(1): 52-59, doi: 10.1111/j.1423-0410.2005.00585.x, indexed in Pubmed: 15663723.

11. Audrain M, Martin J, Fromont P, et al. Autoimmune neutropenia in children: analysis of 116 cases. Pediatr Allergy Immunol. 2011; 22(5): 494-496, doi: 10.1111/j.1399-3038.2010.01117.x, indexed in Pubmed: 21771084.

12. Heusinkveld LE, Majumdar S, Gao JL, et al. WHIM syndrome: from pathogenesis towards personalized medicine and cure. J Clin Immunol. 2019; 39(6): 532-556, doi: 10.1007/s10875-019-00665-w, indexed in Pubmed: 31313072.

13. Majumdar S, Murphy PM. Adaptive immunodeficiency in WHIM syndrome. Int J Mol Sci. 2018; 20(1), doi: 10.3390/ijms20010003, indexed in Pubmed: 30577453.

14. Lewandowski $K$. Future has come: next-generation sequencing in the diagnostics of orphan diseases. Acta Haematol Pol. 2020; 51(4): 191-192, doi: 10.2478/ahp-2020-0034.

15. Rupa-Matysek J, Gil L, Mozer-Lisewska I, et al. Neutropenia - there are always two sides to a story. Acta Haematol Pol. 2020; 51(3): 133-141, doi: 10.2478/ahp-2020-0025.

16. Vlacha V, Feketea G. The clinical significance of non-malignant neutropenia in hospitalized children. Ann Hematol. 2007; 86(12): 865-870, doi: 10.1007/s00277-007-0346-y, indexed in Pubmed: 17653547.

17. Celkan T, Koç BŞ. Approach to the patient with neutropenia in childhood. Turk Pediatri Ars. 2015; 50(3): 136-144, doi: 10.5152/TurkPediatriArs.2015.2295, indexed in Pubmed: 26568688.

18. Angelino G, Caruso R, D'Argenio P, et al. Etiology, clinical outcome, and laboratory features in children with neutropenia: analysis of 104 cases. Pediatr Allergy Immunol. 2014; 25(3): 283-289, doi: 10.1111/ pai.12177, indexed in Pubmed: 24325465.

19. Pick AM, Nystrom KK. Nonchemotherapy drug-induced neutropenia and agranulocytosis: could medications be the culprit? J Pharm Pract. 2014; 27(5): 447-452, doi: 10.1177/0897190014546115, indexed in Pubmed: 25124379. 